\title{
Apéry's Double Sum is Plain Sailing Indeed
}

\author{
Carsten Schneider* \\ Research Institute for Symbolic Computation \\ J. Kepler University Linz \\ A-4040 Linz, Austria \\ Carsten.Schneider@risc.uni-linz.ac.at
}

Submitted: Dec 12, 2006; Accepted: Jan 19, 2007; Published: Jan 29, 2007

Mathematics Subject Classification: 65B10,33F10,68W30

\begin{abstract}
We demonstrate that also the second sum involved in Apéry's proof of the irrationality of $\zeta(3)$ becomes trivial by symbolic summation.
\end{abstract}

In his beautiful survey [4], van der Poorten explained that Apéry's proof [1] of the irrationality of $\zeta(3)$ relies on the following fact: If

$$
a(n)=\sum_{k=0}^{n}\left(\begin{array}{c}
n+k \\
k
\end{array}\right)^{2}\left(\begin{array}{l}
n \\
k
\end{array}\right)^{2}
$$

and

$$
b(n)=\sum_{k=0}^{n}\left(\begin{array}{c}
n+k \\
k
\end{array}\right)^{2}\left(\begin{array}{l}
n \\
k
\end{array}\right)^{2}\left(H_{n}^{(3)}+\sum_{m=1}^{k} \frac{(-1)^{m-1}}{2 m^{3}\left(\begin{array}{c}
n+m \\
m
\end{array}\right)\left(\begin{array}{c}
n \\
m
\end{array}\right)}\right)
$$

where $H_{n}^{(3)}=\sum_{i=1}^{n} \frac{1}{i^{3}}$ are the harmonic numbers of order three, then both sums $a(n)$ and $b(n)$ satisfy the same recurrence relation

$$
(n+1)^{3} A(n)-(2 n+3)\left(17 n^{2}+51 n+39\right) A(n+1)+(n+2)^{3} A(n+2)=0 .
$$

Van der Poorten points out that Henri Cohen and Don Zagier showed this key ingredient by "some rather complicated but ingenious explanations" [4, Section 8] based on the creative telescoping method.

Due to Doron Zeilberger's algorithmic breakthrough [9], the $a(n)$-case became a trivial exercise. Also the $b(n)$-case can be handled by skillful application of computer algebra: In [10] Zeilberger was able to generalize the Zagier/Cohen method in the setting of

\footnotetext{
${ }^{*}$ Supported by the SFB-grant F1305 and the grant P16613-N12 of the Austrian FWF
} 
WZ-forms. Later developments for multiple sums [8, 7] together with holonomic closure properties [5, 3] enable alternative computer proofs of the $b(n)$-case; see, e.g., [2].

Nowadays, also the $b(n)$-case is completely trivialized: Using the summation package Sigma [6] we get plain sailing - instead of plane sailing, cf. van der Poorten's statement in [4, Section 8]. Namely, after loading the package into the computer algebra system Mathematica

\section{$\ln [1]:=<$ Sigma.m}

Sigma - A summation package by Carsten Schneider (c) RISC-Linz

we insert our sum mySum $=b(n)$

$$
\ln [2]:=\operatorname{mySum}=\sum_{k=0}^{n}\left(\begin{array}{c}
n+k \\
k
\end{array}\right)^{2}\left(\begin{array}{l}
n \\
k
\end{array}\right)^{2}\left(H_{n}^{(3)}+\sum_{m=1}^{k} \frac{(-1)^{m-1}}{2 m^{3}\left(\begin{array}{c}
n+m \\
m
\end{array}\right)\left(\begin{array}{c}
n \\
m
\end{array}\right)}\right) ;
$$

and produce the desired recurrence with

\section{$\ln [3]:=$ GenerateRecurrence[mySum]}

$$
\text { Out }[3]=\left\{(n+1)^{3} \operatorname{SUM}[n]-(2 n+3)\left(17 n^{2}+51 n+39\right) \operatorname{SUM}[n+1]+(n+2)^{3} \operatorname{SUM}[n+2]==0\right\}
$$

where $\operatorname{SUM}[n]=b(n)=$ mySum. The correctness proof is immediate from the proof certificates delivered by Sigma.

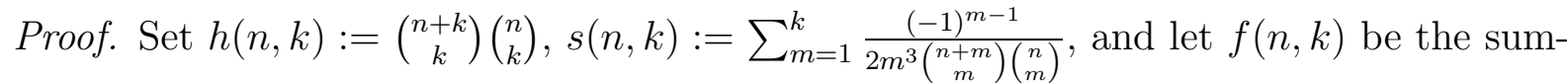
mand of (1), i.e., $f(n, k)=h(n, k)^{2}\left(H_{n}^{(3)}+s(n, k)\right)$. The correctness follows by the relation

$$
s(n+1, k)=s(n, k)-\frac{1}{(n+1)^{3}}-\frac{(-1)^{k-1}}{(n+1)^{2}(n+k+1) h(n, k)}
$$

and by the creative telescoping equation

$$
c_{0}(n) f(n, k)+c_{1}(n) f(n+1, k)+c_{2}(n) f(n+2, k)=g(n, k+1)-g(n, k)
$$

with the proof certificate given by $c_{0}(n)=(n+1)^{3}, c_{1}(n)=17 n^{2}+51 n+39, c_{2}(n)=$ $(n+2)^{3}$, and

$$
g(n, k)=\frac{h(n, k)^{2}\left[p_{0}(n, k) H_{n}^{(3)}+p_{1}(n, k) \sum_{m=1}^{k} \frac{(-1)^{m-1}}{2 m^{3}\left(\begin{array}{c}
n+m \\
m
\end{array}\right)\left(\begin{array}{c}
n \\
m
\end{array}\right)}\right]+(-1)^{k} h(n, k) p_{2}(n, k)}{(n+1)^{2}(n+2)(-k+n+1)^{2}(-k+n+2)^{2}}
$$

where

$$
\begin{aligned}
p_{0}(n, k)= & 4 k^{4}(n+1)^{2}(n+2)(2 n+3)\left(2 k^{2}-3 k-4 n^{2}-12 n-8\right), \\
p_{1}(n, k)= & 4 k^{4}(n+1)^{2}(n+2)(2 n+3)\left(2 k^{2}-3 k-4 n^{2}-12 n-8\right), \\
p_{2}(n, k)= & k(k+n+1)(2 n+3)\left(-8 n^{4}+24 k n^{3}-48 n^{3}-31 k^{2} n^{2}+109 k n^{2}\right. \\
& \left.-104 n^{2}+13 k^{3} n-100 k^{2} n+159 k n-96 n+21 k^{3}-81 k^{2}+74 k-32\right) .
\end{aligned}
$$

Relation (3) is straightforward to check: Take its shifted version in $k$, subtract the original version, and then verify equality of hypergeometric terms. To conclude that (4) holds for 
all $0 \leq k \leq n$ and all $n \geq 0$ one proceeds as follows: Express $g(n, k+1)$ in (4) in terms of $h(n, k)$ and $s(n, k)$ by using the relations $h(n, k+1)=\frac{(n-k)(n+k+1)}{(k+1)^{2}} h(n, k)$ and $s(n, k+1)=s(n, k)+\frac{(-1)^{k}}{2(k+1)^{3} h(n, k+1)}$. Similarly, express the $f(n+i, k)$ in (4) in terms of $h(n, k)$ and $s(n, k)$ by using the relations $h(n+1, k)=\frac{n+k+1}{n-k+1} h(n, k)$ and (3). Then verify (4) by polynomial arithmetic. Finally, summing (4) over $k$ from 0 to $n$ gives Out[3] or $(2)$.

In conclusion, we remark that the harmonic numbers $H_{n}^{(3)}$ in (1) are crucial to obtain the recurrence relation (2). More precisely, for the input sum

$$
\sum_{k=0}^{n}\left(\begin{array}{c}
n+k \\
k
\end{array}\right)^{2}\left(\begin{array}{l}
n \\
k
\end{array}\right)^{2} \sum_{m=1}^{k} \frac{(-1)^{m-1}}{2 m^{3}\left(\begin{array}{c}
n+m \\
m
\end{array}\right)\left(\begin{array}{c}
n \\
m
\end{array}\right)}
$$

Sigma is only able to derive a recurrence relation of order four.

\section{References}

[1] R. Apéry. Irrationalité de $\zeta(2)$ et $\zeta(3)$. Astérisque, 61:11-13, 1979.

[2] F. Chyzak. Variations on the sequence of Apéry numbers. http://algo.inria.fr/libraries/autocomb/Apery-html/Apery.html.

[3] C. Mallinger. Algorithmic manipulations and transformations of univariate holonomic functions and sequences. Master's thesis, RISC, J. Kepler University, Linz, 1996.

[4] A. van der Poorten. A proof that Euler missed... Apéry's proof of the irrationality of $\zeta(3)$. Math. Intelligencer, 1:195-203, 1979.

[5] B. Salvy and P. Zimmermann. Gfun: A package for the manipulation of generating and holonomic functions in one variable. ACM Trans. Math. Software, 20:163-177, 1994.

[6] C. Schneider. Symbolic summation assists combinatorics. Sém. Lothar. Combin., 56:1-36, 2007. Article B56b.

[7] K. Wegschaider. Computer generated proofs of binomial multi-sum identities. Diploma thesis, RISC Linz, Johannes Kepler University, 1997.

[8] H. Wilf and D. Zeilberger. An algorithmic proof theory for hypergeometric (ordinary and "q") multisum/integral identities. Invent. Math., 108:575-633, 1992.

[9] D. Zeilberger. The method of creative telescoping. J. Symbolic Comput., 11:195-204, 1991.

[10] D. Zeilberger. Closed form (pun intended!). Contemp. Math., 143:579-607, 1993. 